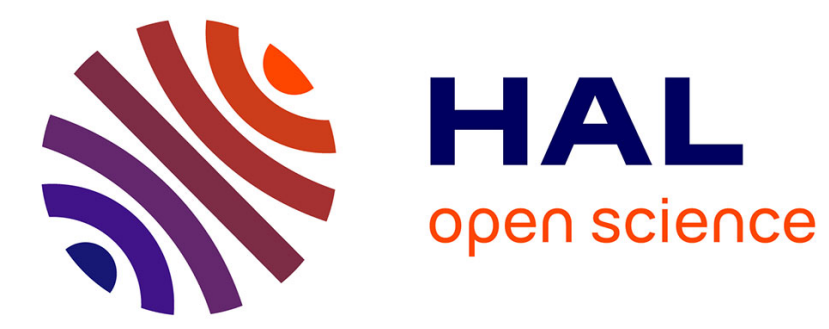

\title{
Communicating Data to an Audience
}

Steven M. Drucker, Samuel Huron, Robert Kosara, Jonathan Schwabish, Nicholas Diakopoulos

\section{To cite this version:}

Steven M. Drucker, Samuel Huron, Robert Kosara, Jonathan Schwabish, Nicholas Diakopoulos. Communicating Data to an Audience. Data-Driven Storytelling, 2019, 9781315281568. hal-02310515

\section{HAL Id: hal-02310515 https://hal.science/hal-02310515}

Submitted on 10 Oct 2019

HAL is a multi-disciplinary open access archive for the deposit and dissemination of scientific research documents, whether they are published or not. The documents may come from teaching and research institutions in France or abroad, or from public or private research centers.
L'archive ouverte pluridisciplinaire HAL, est destinée au dépôt et à la diffusion de documents scientifiques de niveau recherche, publiés ou non, émanant des établissements d'enseignement et de recherche français ou étrangers, des laboratoires publics ou privés. 


\section{Communicating Data to an Audience}

Steven Drucker, Samuel Huron, Robert Kosara, Jonathan Schwabish, Nicholas Diakopoulos

\section{Introduction}

Communicating data in an effective and efficient story requires the content author to recognize the needs, goals, and knowledge of the intended audience. Do we, the authors, need to explain how a particular chart works? It depends on the audience. Does the data need to be traced back to its source? Depends on the audience. Can we skip obvious patterns and correlations and dive right into the deeper points? Depends on the audience. Do we need to explain what the findings in the data mean in terms of what the data represents? Depends on the audience. There are many more questions for which this is true.

It appears reasonable, then, to know who that audience is and what they know. In addition, we might also want to know what their expectations and needs are: What does the audience want to get out of the story? A single story cannot possibly address all possible different audiences and their needs. And any well-designed story will be tailored not just to its data and the intended message, but to its audience.

That is the theory, at least. In practice, this is quite difficult to achieve. A large audience will consist of people with different backgrounds and knowledge levels that are impossible to target at the same time; in breaking news and media production, short deadlines often make it impractical to create multiple versions of a news graphic that will work on different devices; in other fields, personnel or financial considerations may constrain the ability to create a product that targets the correct audience. Knowledge about the audience is also often quite limited. It tends to be general and broad, and usually not specific enough to target visualizations to individuals.

Despite these limitations, considering the audience, even broadly, can help guide the design of visual stories. The results are more appealing, effective, and meaningful to the intended recipients.

In some cases, content authors know precisely who a presentation or report will be given to: what they know about the data, the background, etc. In other cases, the story author may at least have an expectation or an imagination of who they think the audience may be (Litt, 2012), such as the general level of data and visualization literacy of a particular publication's target audience. Such audience expectations inform the editorial decisions that need to be made about visualizations and news graphics. For instance, if the intended audience for a story is teenagers with limited statistical or visualization literacy, then additional explanation or context might be needed for less common chart types so that they can be accurately interpreted. 
Different audiences not only have different levels of familiarity and literacy with visualization, they also have different goals, expectations, attention spans, and cognitive abilities. A general reader of a newspaper has very different needs and goals than an academic researcher reading a scholarly journal or a policymaker reading a briefing memo. A television audience will expect a different kind of presentation than a newsprint reader or a news website user, who in turn will have different expectations than those in a meeting with colleagues.

A television audience's attention span might be on the order of a few seconds. For web audiences, some data show that 55 percent of users spend less than 15 seconds on an item of news content (Haile, 2014). By contrast, communicating data and stories to people who need the information to make decisions or change policies - for example, executive managers or policymakers - attention may be substantially longer because the information being communicated is essential to their performance. Other audiences may want to accomplish different things by reading a data story, such as being entertained, educated, or satisfying their general curiosity.

To effectively communicate ideas and concepts, content authors need to think carefully about how their work best fits the needs of the audience. In this chapter, we explore design considerations relating to audience knowledge and goal contexts, and consider the difference between the theory of what we might know and the reality of what we can know. We discuss some approaches that allow us to tailor a piece to the audience with little or no knowledge about them. Finally, we describe a few specific design contexts and their particular requirements, such as news graphics and broadcast television.

\section{What Does the Audience Know?}

Identifying the background knowledge of an audience for any given project is a challenging endeavor. In this section we identify some of the audience knowledge characteristics that are important when communicating information driven by data and told through text, images, and data visualizations.

Through discussion of our experiences coming from different backgrounds and past work experiences, we have identified three general areas where content producers could know more about their audience. Such knowledge would enable more effective communication of data-driven content.

1. How literate is the audience in terms of data and visualization? And what can be done to increase it? The answer in news graphics tends to be an annotation layer.

2. How knowledgeable is the audience in terms of jargon, domain expertise, and other background knowledge?

3. What are audience expectations about the design of visualization, such as style, tone, or use of iconography? 


\section{Data and Visualization Literacy: The Annotation Layer}

Definitions of visualization literacy describe the ability to interpret visual patterns of the underlying data, and to confidently use a visualization to answer questions about the data (Boy et al, 2014). As the value and availability of data continues to grow, so does the demand for understanding data and graphs -- of increasing visualization literacy. Data science and data visualization courses and training in the private sector, in post-secondary schools, and in online training programs have increased substantially over the past few years (Womack, 2014). The onus isn't just on the audience though, data storytellers have an ethical obligation to ensure that their visualizations do not skew or mislead interpretations unnecessarily (see Chapter 10: "Ethics in Data-Driven Visual Storytelling"), and this intersects with the literacy level of the expected audience. Increasing an audience's understanding of different data and graph types may come from a variety of sources, some within the content-producers' control, others not.

In news graphics, the solution is what is usually called the annotation layer It adds explanations and descriptions to introduce the graph's context, which is important for almost any audience. It also explains how to read the graph, which helps readers unfamiliar with the graph - whether a simple line chart or an advanced technique like a treemap or scatterplot. When done right, it will not get in the way for experienced ones.

Annotations and explanations have a long history. Figure 9.1 shows a line chart in The New York Daily Tribune in 1849 in which the addition of an explanatory caption and annotations help guide the interpretation of how to read the then still unfamiliar line chart. 


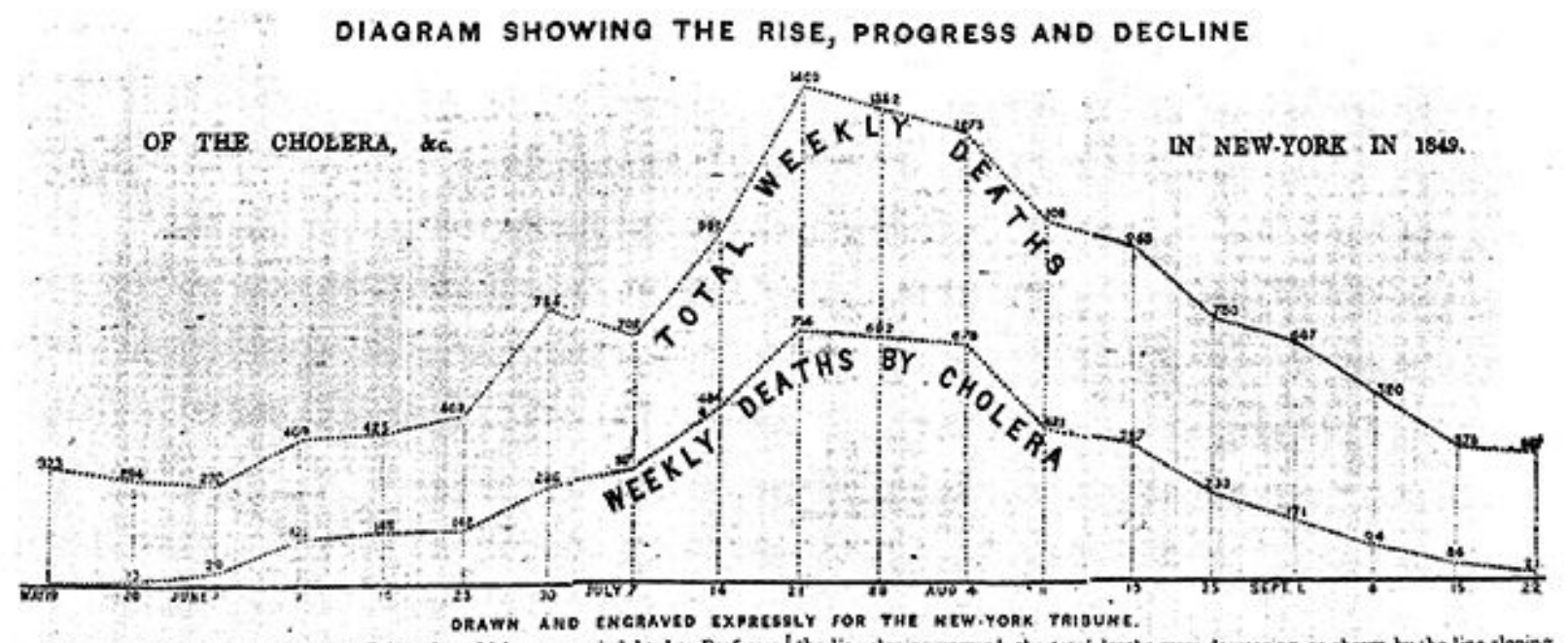

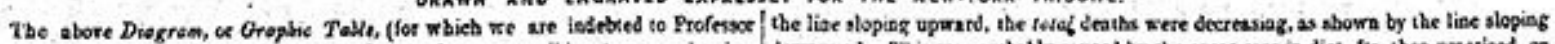

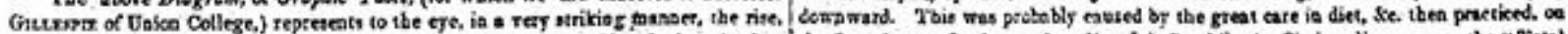

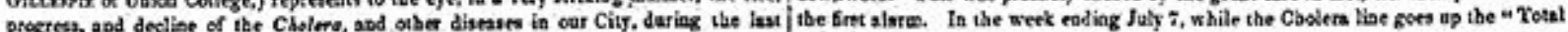

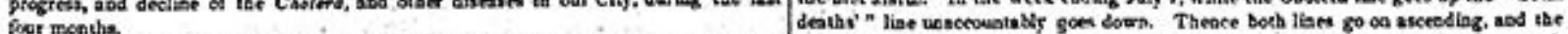

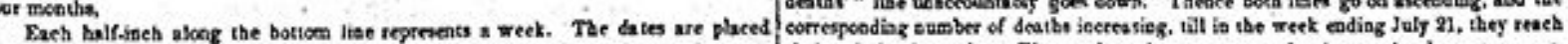

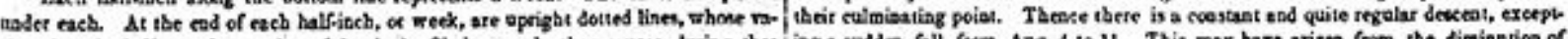

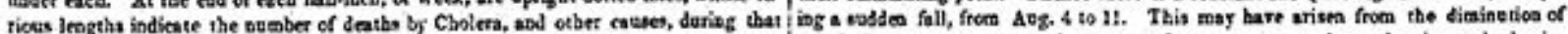

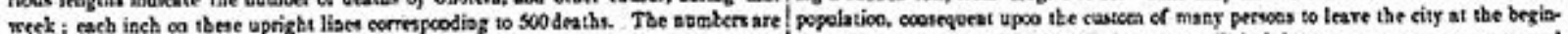

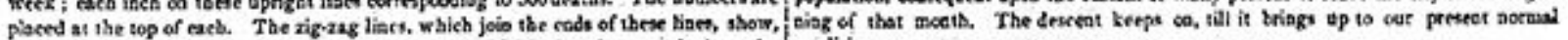
if theit apwasd of dowaward slopes, whethet the deathe deriaz those wieks bave ib oceditios.

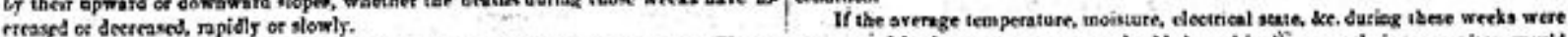

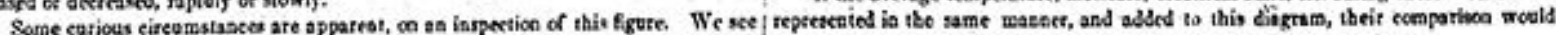

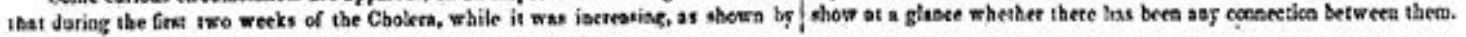

Figure 9.1. A line chart printed in The New York Daily Tribune, September 29, 1849 would not have been familiar to many of the newspaper's readers. As a result, extensive instructions are given for how to decode and understand the chart.

Amanda Cox, the editor of the Upshot at the New York Times, is famously quoted as saying, "The annotation layer is the most important thing we do...otherwise, it's a case of here it is, you go figure it out" (Cox, 2011).

Annotation goes beyond just labeling points or lines on a chart: the bubble plot shown in Figure 9.2 from the Los Angeles Times, for example, expertly combines annotation that explains how to read the chart with what the content means. The chart shows the relationship between the change in violent crime rate (horizontal axis) and the property crime rate (vertical axis) in about 30 cities in California. For readers familiar with this chart type, it is immediately clear how to glean conclusions from the data. The average LA Times reader may not be familiar with this chart type, so upon first viewing the graphic, there is a big, red box in the top-right with big, red text that says "Worse" and a big, blue box in the bottom-left with big, blue text that says "Better". Then, each quadrant has a small headline in boldface text with a sentence below to deliver the content. Thus, even for the reader who has never seen a bubble plot before, the annotation instructs them on how to read the chart, and then delivers the content through the additional explanatory text. 


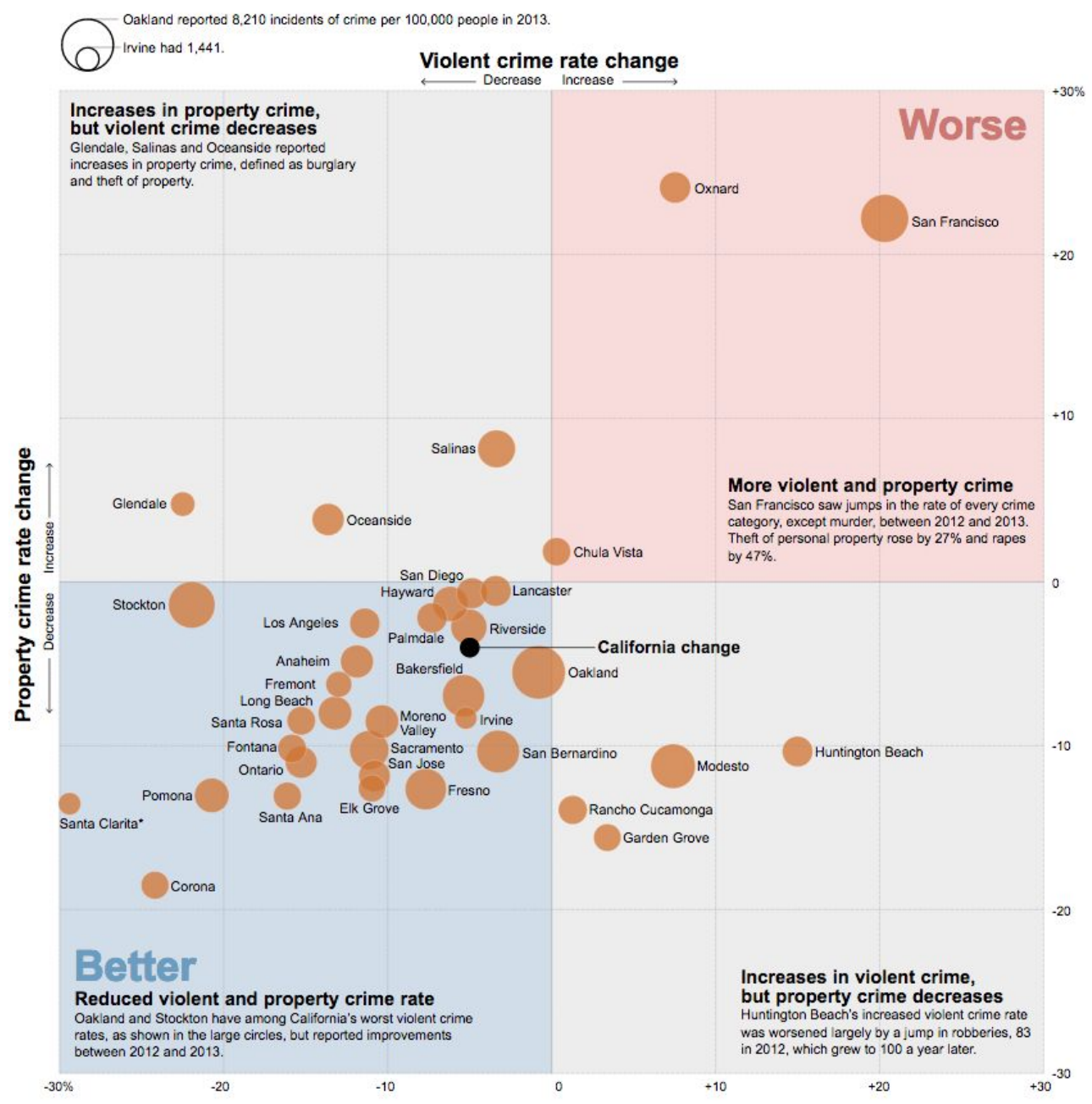

Figure 9.2. A bubble plot from the Los Angeles Times from 2013 expertly annotates both how to read the chart and the story the authors deliver. Source: http://graphics.latimes.com/california-crime-2013/

\section{Background Knowledge and Expertise}

A content-producer may face an audience with different levels of expertise or knowledge on any given topic. Readers of a large daily newspaper like the New York Times or Washington Post may reflect a variety of different levels of expertise, especially across the different topics covered by the publication. By contrast, readers of the Financial Times may have more domain-specific expertise in certain areas, especially as the Financial Times tends to focus their reporting on the financial sector. The audience for a small local publication is likely to have knowledge of the local area, whereas the same couldn't be assumed for the audience of a large national or international publication. Data storytelling needs to take into account the baseline of information and knowledge that the intended audience is expected or assumed to have. 
Different levels of expertise and knowledge should encourage content-producers to think carefully about how their products will best meet the needs of a diverse audience. Niche audiences may be more comfortable with the jargon of their group, but designers may consider whether removing jargon would serve a larger audience just as easily while making the content more broadly accessible. Universal design is an aspiration that can allow data stories to appeal to and be used by a broad array of people (Shneiderman et al, 2016), but design is also about tradeoffs that may make a data story less appealing to some, while simultaneously much more appealing and useful to others. In such cases different versions of the story might be produced for different audiences, each targeting a unique range of knowledge, expertise, and other factors discussed in this chapter.

An individual in the audience brings their own viewpoints, backgrounds, and experience to each and every data-driven story they consume. Whether driven by their cultural background, social position, education, or other demographic characteristics, readers carry with them their own unique set of knowledge and biases. While it is impossible for content-producers to be aware of each individual's particular experience--to know in advance what they already know--it may be possible to group individuals (i.e. to segment the audience) in ways that are useful to guiding design.

Some examples may be illustrative here. The first comes from the Congressional Budget Office (CBO), which is the budget arm of the U.S. Congress. CBO reports and numbers are regularly used and referenced by Congress, as is often dictated by law. Their audience of Members of Congress and their staffs is well-defined and well-known. That audience wants (and needs) headline numbers, facts, and statistics that they can use to communicate with their colleagues and their constituents. In June 2012, CBO published its Long-Term Budget Outlook, a 109-page report about the budget outlook for the federal government (CBO 2012). Paired with that report was a one-page infographic that highlighted the top-level items, facts, and patterns (see Figure 9.3). In a congressional hearing about the long-term budget outlook, Ranking Member Congressman Chris van Hollen (D-MD) held up that infographic as an exhibit to support his point. From the perspective of $\mathrm{CBO}$, this is arguably recognizing the needs of their audience and how a member of Congress could effectively use their analysis. 


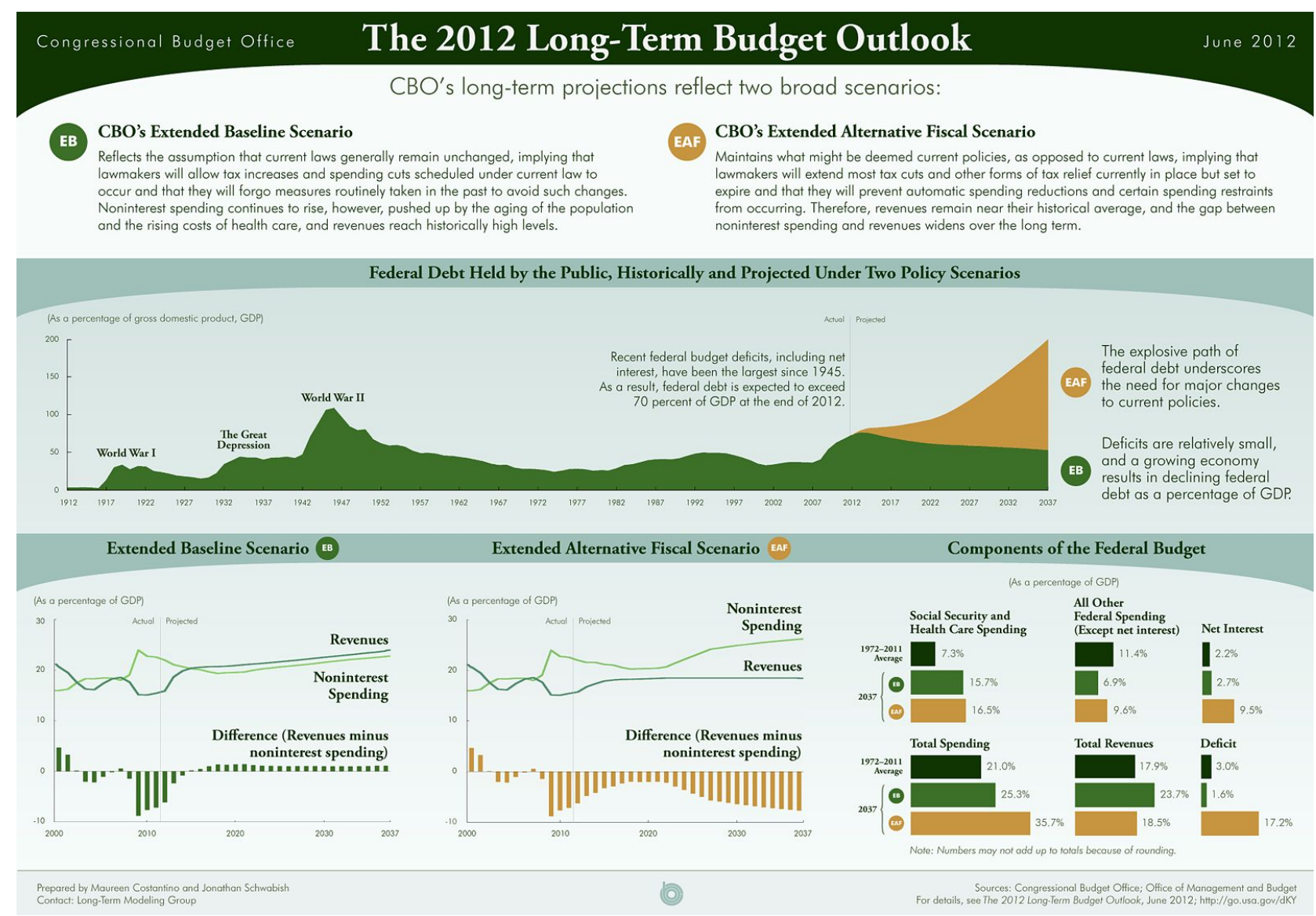

Figure 9.3. The 2012 Long-Term Budget Outlook infographic from the Congressional Budget Office. Source: https://www.cbo.gov/publication/43289

Another example comes from the Urban Institute, a nonprofit research organization in Washington, DC where one of the authors currently works. The Urban Institute is interested in communicating with policymakers, but is also interested in communicating their work to a broader audience of local and state policymakers, other researchers, practitioners, the press, and the public more broadly (see, also, Chapter 8: "Organizing the Work of Data-Driven Visual Storytelling"). Thus, in some ways, Urban is similar to the news organizations mentioned above, but communicating to an audience that is particularly interested in the kind of in-depth social and public policy research they conduct.

In August 2013, with the release of a report on the potential policy responses to long-term unemployment in the U.S., Urban published a "long form narrative" story on its website that combined the highlights from the report with interactive data visualizations, photographs, and sound clips from interviews with people who were unemployed (Acs, 2013). Recognizing that the traditional reports may not always have impact or reach a broad(er) audience, this type of data-driven story--which is now common for news organizations--is (still) fairly uncommon in the social science and non-profit research space. 


\section{Design Expectations}

Basic data visualization best practices and strategies are needed across multiple domains to help content-creators find better ways to communicate their work to their audiences. Many people who have experience working with data and conducting in-depth analysis may not have the knowledge or experience in how to communicate their work. The field of data visualization--a broad field that can be considered to encompass data journalism, data science, data visualization scientific research, as well as general practitioners--continues to evolve in the face of new methods, tools, and data. A growing number of books, blogs, and articles have been published just over the past few years dedicated to helping people improve their visualizations.

A consistent design approach (one might call it "visual branding") can help an audience relate to a producer's work and entice them to return. Consistency across color, font, layout, and type of interactivity, for example, can help the audience better identify what the producer is going to deliver, when, and for what level of expertise. Conventions used in presentation can also reinforce good data hygiene and inform the audience as to the quality of a visualization such as when source and designer credits or methodologies are consistently incorporated into the story delivery (Hullman and Diakopoulos, 2011; Schwabish, 2016a).

Graphics from outlets such FiveThirtyEight, Vox, New York Times, The Economist, and others each have a specific design aesthetic. The inclusion of their brands' colors and fonts, a specific text or annotation style, and method of publication (e.g., daily, blogs, etc.) all help audiences recognize and relate to the producer's content. Practically, a design aesthetic is enculturated by having style guides that designers working for a given publication use to help choose colors, fonts, and layout. Non-profit organizations such as the Urban Institute ${ }^{1}$ (See Figure 9.4) as well as news outlets such as the Dallas Morning News ${ }^{2}$ have even published their style guides publicly. These guides help enforce a consistency of visual output (see Schwabish 2016b for a collection of other style guides).

\footnotetext{
${ }^{1}$ http://urbaninstitute.github.io/graphics-styleguide/

${ }^{2}$ https://knightcenter.utexas.edu/mooc/file/tdmn_graphics.pdf
} 


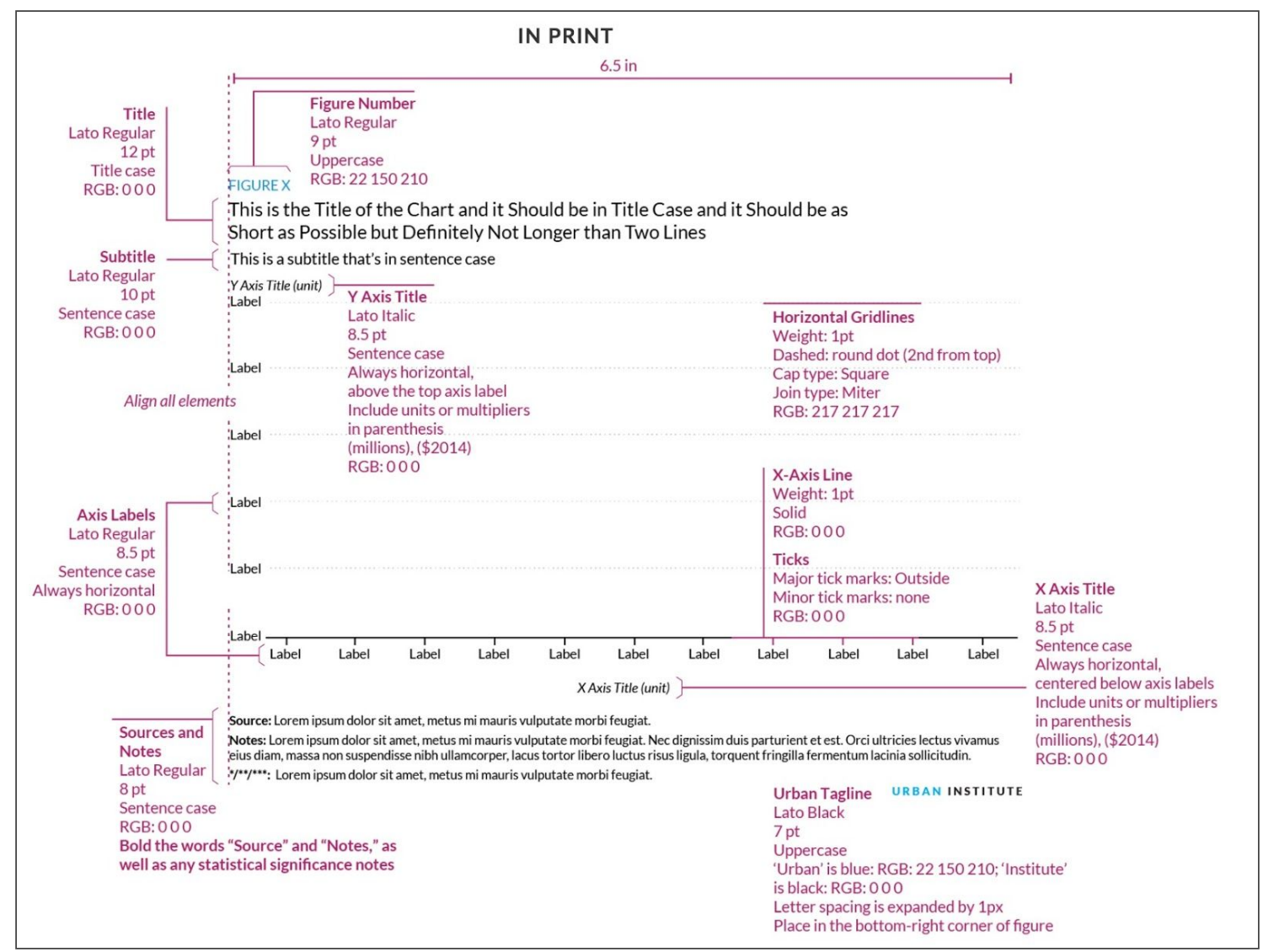

Figure 9.4. From the "Chart Parts" section of the Urban Institute Data Visualization Style Guide. Source: http://urbaninstitute.github.io/graphics-styleguide/

Another dimension that informs design expectations are cultural differences and viewing codes. For instance the layout of design elements such as the title and credits in Western cultures may not meet expectations in cultures that read from right to left. Such differences may be particularly relevant for complex designs that can evoke a layer of meaning via connotation. For instance, colors can suggest very different meanings in the east versus west: in Asia white is often the color for mourning, whereas it is black in most of the west (Ware, 2008). This complicates the design process for data stories that are oriented towards a global audience such as may be the case for international publications like The Economist.

\section{What Does the Audience Want?}

Like any other designed experience, data stories are more impactful and useful when they are crafted with the motivations, goals, and tasks of users in mind -- in this case the audience for the story. The following subsections explore several typical wants and needs from media consumption that may impact design, as well how interactivity can be leveraged to increase the adaptability of data stories to different user needs and contexts. 


\section{Media Wants and Needs}

The uses and gratifications framework describes a set of dimensions that can influence why people consume media (Ruggiero, 2000). The idea is that people receive certain gratifications from the media they consume that help satisfy various underlying social or psychological needs. For instance people may seek out data-driven stories for a variety of reasons such as to inform themselves on an issue of personal (e.g., health) or civic importance (e.g., elections), or to casually lean back and be entertained or pass time in a non goal-oriented fashion. Uses and gratifications theory suggests that there are four primary underlying motivations for media consumption:

(1) staying informed about current information, satisfying curiosity and self-education;

(2) gaining insight into oneself or seeing models for others' behavior;

(3) socially interacting and connecting with others, or as a basis for social conversation, or developing empathy; and

(4) as relaxing entertainment or diversion.

A data-driven story can be designed differently depending on whether the audience is expected to approach the experience from a more social, entertainment, or strictly informational motivation. An entertaining treatment might take more license with visual novelty and not focus as closely on efficiency in decoding visual information, for example. A social treatment might emphasize user comments and responses to a data story, whereas an information-oriented design may focus on well-researched and validated background context provided in an annotation layer. The goal as a designer is to explore the design space in order to try to match the data story to the expected media need of the audience.

An individual's needs from a piece of media like a data story can also vary with the intended interface. For instance, if a story is likely to be consumed on a mobile device, the user could be in a loud or distracting environment that might undermine the use of audio in the narrative (written annotations as backup is a nice design strategy). The specific location of a user on a GPS-enabled device can be interesting context that can be used to inform the way a story is presented (Kim, Hullman, Agrawala, 2016). In contexts that are more casual than work-oriented, users may have a higher degree of interruptibility, which could mean that a data story is consumed in short bursts of attention. Presenting information in "chunked" patterns might be more effective than as a single, long-form narrative in such cases. Finally, it's worth considering that boredom may be a driving factor for interaction in contexts where the user is consuming a data story while waiting (e.g., for a bus or train), or splitting attention between devices and screens (e.g., as many people do when watching television). 


\section{Tailoring to the Audience Without Knowing It}

Even if we don't know anything about the audience, we can make it appear as if we did. The audience knows itself: they can pick their city in a story about home prices, their age and gender in a story on health, and their education and income levels in a story about taxes or inequality.

This self-identification in a visualization may be why maps are such a popular tool in data visualization. While maps can often present data inaccurately, or even mislead a reader, our familiarity with maps makes them a convenient and familiar visualization choice (Wiseman 2015). Alternatives--such as cartograms, which distort geographic areas to correspond to the data values--sometimes have the advantage of portraying the data in more accurate ways, but the distortion makes the visualization less familiar to the reader.

A variety of other projects and research have tested or demonstrated the importance of allowing the audience to "find themselves" or make content more familiar in a visualization. For example, research by Chevalier, Vuillemot, and Gali (2013) attempt to build a framework to help people think about the best ways to communicate large numbers. More specifically, Kim, Hullman, and Agrawala (2016) construct an online interactive application that enables users to re-express spatial measurements in localized terms--in other words, users are able to build a map that makes a distance relevant to their area or experience.

Even outside of maps, readers locating themselves in the data is likely to make them more engaged. A piece breaking down the unemployment rate by race, gender, age group, and education level (Carter et al, 2009) allows the reader to compare themselves to the average and to other specific groups. While not all possible criteria are covered, this piece gets much closer to an individual than just the unemployment rate that averages across everybody. Similarly, a line chart showing the Case-Shiller Home Price Index for 20 cities lets the reader pick their city and see how housing prices have changed over the years (Carter and Quealey, 2014).

\section{Directly Engaging the Audience}

A somewhat similar approach asks the audience questions to involve them in what is shown. This can help engagement by allowing them to place themselves in a context (and at the same time also collect data to be shown to everybody), and it has recently also been found to help people remember what they have seen.

In 2011, The New York Times published The Death of a Terrorist: A turning point? (Huang 2011) where readers were invited to position their opinions on the death of Osama bin Laden in a two dimensional matrix: whether their response was positive or negative overall, and whether they thought that this event was going to be significant in the war on terror or not. The resulting heatmap of responses showed the landscape of people's responses. 
Similar pieces have since appeared in different media, such as the Times' You Draw It: How Family Income Predicts Children's College Chances, which allowed users to draw their idea of the correlation between parents' income and their children's ability to attend college (Aisch et al, 2015). Similar pieces have appeared to let readers predict price changes for common goods to match an inflation goal (Canipe, 2016), draw the border between Western Germany and the German Democratic Republic from memory (Keseling, 2015), or guess the rate of teenage pregnancies in the UK (ONS, 2016) (Figure 9.5).

\section{For every 100 girls aged under 18 in 2014, how many became pregnant?}

This is all conceptions for girls under 18 , per 100 girls aged 15 to 17 .

Use the slider to make your guess

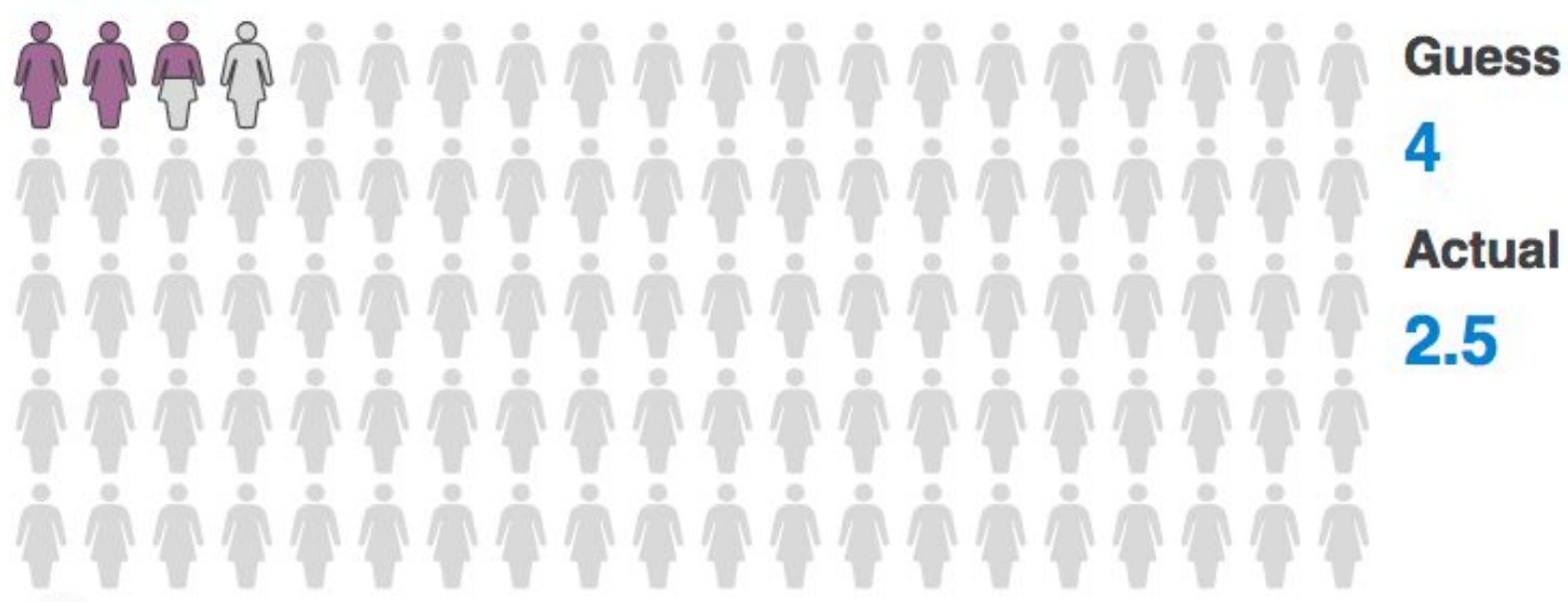

\section{You're not too far away but you've gone a bit too high!}

Figure 9.5. In this interactive piece by the UK's Office of National Statistics, readers are first asked to make an estimate using the slider at the bottom, which fills in as many people as they are guessing (girls under 18 becoming pregnant, in this case). When they submit their guess, the difference between it and the real value is illustrated by a small animation and the numbers are shown on the right (ONS, 2016).

Interactive pieces like this are still relatively rare, but they are slowly gaining momentum. A study recently tested the effect of asking readers to make estimates before showing the actual data on memory (Kim et al, 2017) (Figure 9.6). It showed that asking participants to reflect on their prior knowledge by guessing numbers or explaining them helped readers to with both comprehension and memory of the data.

Engaging an audience is possible even without much knowledge about its members' backgrounds, level of domain knowledge, etc. Personalization as discussed in the previous section and engagement through questions have both been tried and found to be quite effective. 


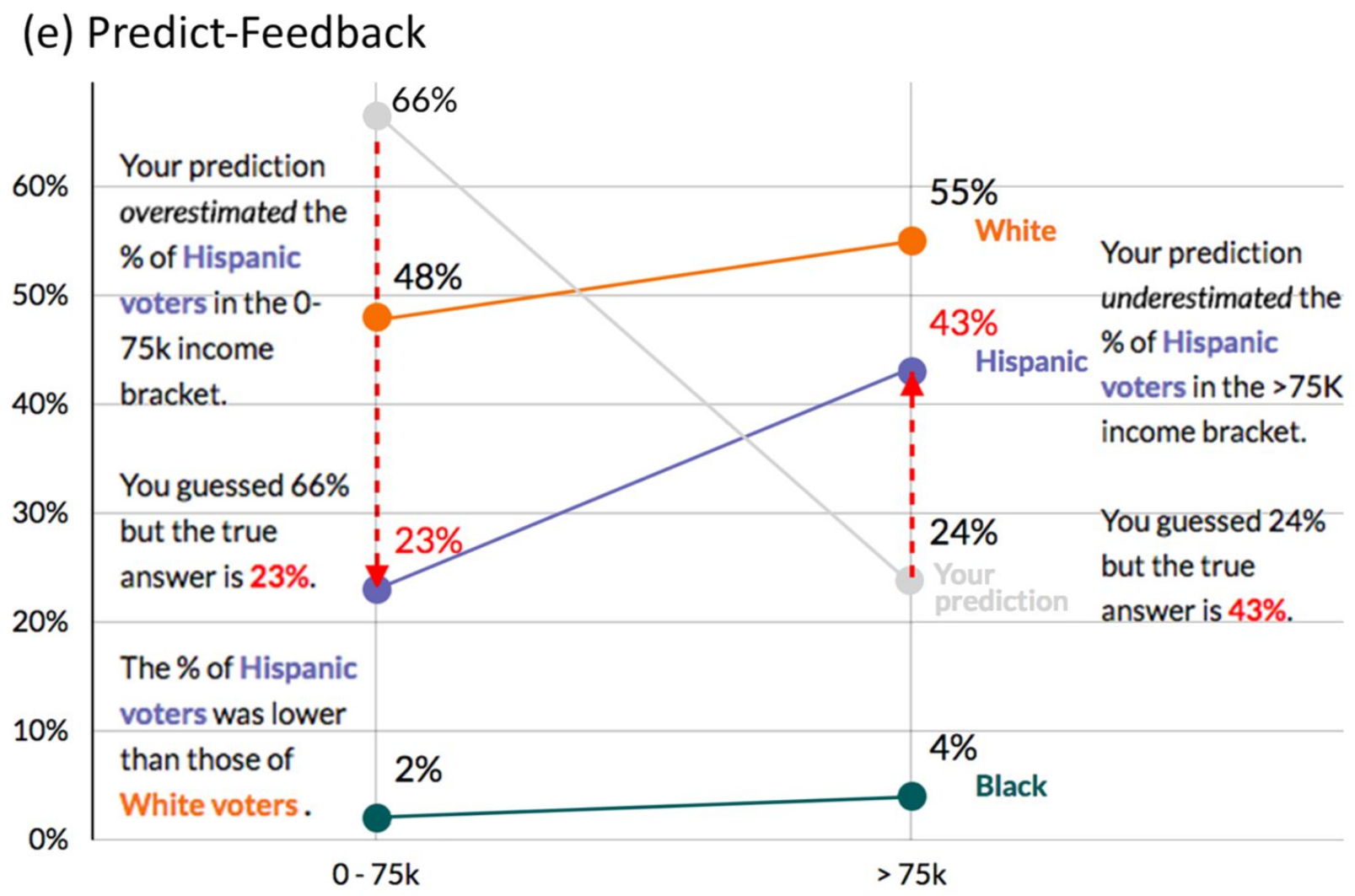

Figure 9.6. User interface used in a study to test the effectiveness of engaging readers by asking them to make guesses about the data first and then showing them how close (or far off) they were (Kim et al, 2017).

\section{Specific Design Contexts}

In addition to the audience itself, the media used to reach it are an important factor that shape what is possible in practice and what people expect. A detailed news graphic designed for print will not work on television, and a TV piece will look out of place on the web.

\section{The Reality of the Newsroom}

Over the last several years news media have created many of the most exciting examples of data-driven storytelling. News media, like other organizations, have struggled with different device types, browser idiosyncrasies, screen sizes, as well as different technologies and programming languages (such as the switch from Flash to HTML 5).

Designing for devices means not only taking their idiosyncrasies into account, but taking advantage of them. Trying to design across many devices typically ends up with a sort of lowest common denominator that works but is not as effective as it could be on any specific device. 
See Chapter 6: "Watches to Augmented Reality: Devices and Gadgets for Data-Driven Storytelling" for more details.

Recently, many newsrooms have scaled back those efforts. New York Times Graphics Editor Gregor Aisch described the problem as a cost-benefit tradeoff (Aisch, 2016). Many interactive news pieces see very little interaction--only $10-15 \%$ of visitors interact with them. Even a simple secondary interaction, like left-right swiping to reveal more information when the main flow of the story is swiping down, is rarely used. This is despite efforts like adding prompts.

Designers have devised a number of workarounds to make large graphical pieces work on small screens. For maps, a convenient one is to zoom the map into the location of the user, since that is likely what they are interested in. However, that often means that there is no way to zoom out to see it in its entirety. Aisch therefore proposes a variation of Shneiderman's famous information-seeking mantra: "Overview first, zoom and filter, then details on demand" (Shneiderman, 1996) for the reality of news graphics on mobile devices: "Details first, no zoom and filter, overview on desktop only."

Newsrooms' resources are limited, and they often produce news stories on tight deadlines (see also Chapter 8: "Organizing the Work of Data-Driven Visual Storytelling"). In light of this, it becomes necessary to consider not just the potential information value, but also the effort to make something interactive when so few readers ever get a benefit from it. Similar to Aisch, Tse (2016) argues that interactive data stories (at least in the newsroom) may not be the best approach. He makes a three-point argument:

1. If you make the reader click or do anything other than scroll, something spectacular has to happen.

2. If you make a tooltip or rollover, assume no one will ever see it. If content is important for readers to see, don't hide it.

3. When deciding whether to make something interactive, remember that getting it to work on all platforms is expensive.

Tse's argument rests on changes in technology, and the changing business structure of the media industry (e.g., advertising efforts, subscription packages, etc.).

It is not clear what the solution is to these issues. One approach is certainly better tools that make it easier to create pieces in the first place and allow the author to target different platforms, either automatically or at least with some support. Another is to show potential interactions with little animations. This has been tried in project such as gapminder, but we are not aware of any studies to show if it such animations would actually increase interaction. Perhaps the most important issue is still training the user: if readers are not used to charts responding and allowing them to interact, they won't try hovering over elements, etc. By creating more interactive pieces, media can train their readers to expect everything to be interactive, and thus increase interaction over time (whether this works or not will have to be studied as well, of course). 
While news media tend to have a general idea of their audience, it tends to be vague and hard to use for practical decision-making. There are still considerable hurdles to using what is known

to target the audience more specifically and precisely. There are many open questions here and ample need (and opportunity) for more research that could help improve the way news pieces are built and how they work.

\section{Visualization for Television}

Television is an unusual use case for visualization, even though its visual nature seems to lend itself well to it. This case study is based on the program Le Grand Webzé, which aired in February 24th, 2012 on the France 5 channel (Huron et al 2013).

A TV audience is highly diverse: almost any age, any level of education, any social position, economic role, and cultural interest is represented. Ratings agencies have been tracking audiences for decades, with age groups starting as young as 4 years old, with segments by age, gender, economic status, and if they are living in urban or rural areas. Television audiences are also fickle, often switching programs and channels within less than a second.

The research and development services of the French National Television Network contacted Huron and his colleagues to create a system that would engage a TV audience with online activity during a TV show. The program is focused on web culture and presents a review of the latest web buzz as well as interviews of famous bloggers or web artists. The goal was to allow viewers to interact with each other and to see a visual representation of that interaction on the program.

The design was made for the medium and the way it is consumed. To show vote tallies, bubbles would drop and form into bar charts (See Fig 9.7.). The bubbles included small images of the people who had voted, making for a selfie moment and improving the visual interest of the graphic. Animation was used throughout to blend in with the always-moving nature of television.

Television presents unique design challenges for visualization: the amount of detail is very limited, there is no interaction, motion and animation are crucial, TV graphics are often three-dimensional, etc. Despite its prevalence in popular culture, television as a medium is still almost completely overlooked in visualization research. 


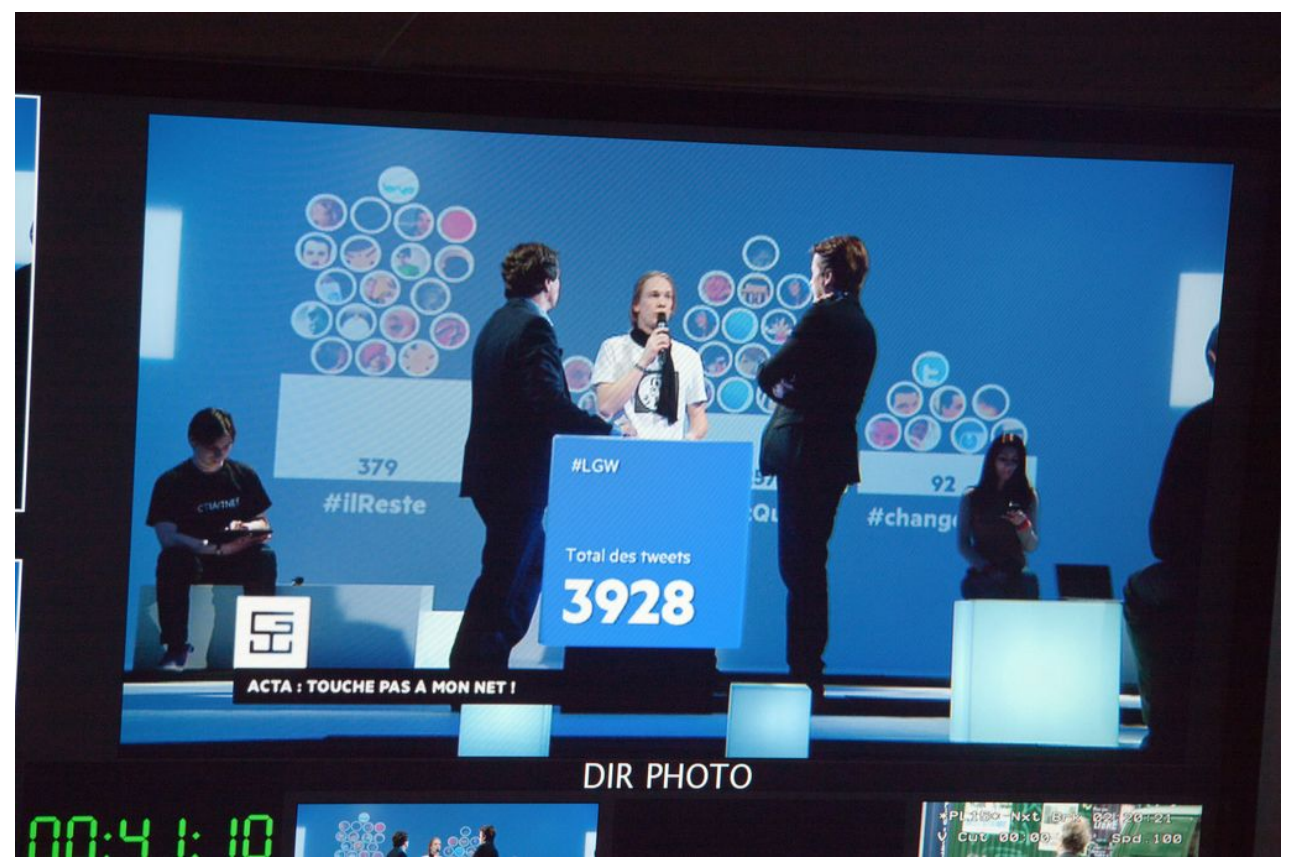

Figure 9.7. Photograph taken of the studio monitoring screen during the Le Grand Webzé TV program. The visualization shows the votes of the show audience about the host's popularity. Each circle represents a tweet sent during the last few seconds, the bar chart represents the total number of tweets for each hashtag. The visualization is updated continuously.

\section{Conclusions}

When presenting data and telling stories, it seems obvious to tailor the presentation to the audience. In practice, unfortunately, there are many reasons why that is not easy or even possible. It could be due to financial or personnel constraints, deadlines, uncertainty about the targeted audience, or even ignorance about audiences capacities. Despite such uncertainty, one way to think about the audience could be to consider their data and visual literacy level, their attention span, background knowledge, and domain expertise.

The inability to know enough of the audience in many cases has led to some interesting approaches that have proven to be helpful beyond just tailoring a visualization to the reader or viewer. Personalization is an effective means not only to tailor a news piece to a particular reader in more detail than could be done otherwise, but also a way to help engage that reader. Asking for the person's background knowledge by having them estimate a value first is necessary when the visualization designer doesn't know the reader, but this has proven helpful with memory and comprehension of the data being shown as well.

In an ideal world, the designer of a data story would know all the relevant information about her audience and be able to put that into practice. Some things are easier to measure or assess, 
like a person's visualization literacy or familiarity with a particular chart type. Others are harder or close to impossible, like a person's background knowledge about a topic or question they might have. Even without being able to answer these questions, a well-designed story brings in many different viewers by providing pieces of context that are likely relevant to most users without cluttering up the display. Going further, it is conceivable that a story would measure a viewer's visualization literacy in an unobtrusive (and clandestine) way and adapt the amount of information being shown, as well as the types of visualization, etc.

Smart design takes available knowledge about the audience into account, but does not depend on it being available. Many design decisions are also driven more by general ideas about effective presentation than specifics about a particular audience. A better understanding of the audience will likely lead to better data stories, but the current state of the art in new graphics, television, and other areas shows that good designers are able to create compelling stories even with barely any knowledge of the audience at all.

\section{References}

Tse, Archie. 2016. "Why We Are Doing Fewer Interactives," presentation at the 2016 Malofiej Infographics World Summit, https://github.com/archietse/malofiej-2016/blob/master/tse-malofiej-2016-slides.pdf.

Aisch, Gregor, 2016. "Data visualization and the news," presentation at the 2016 Information Plus Conference. https://vimeo.com/182590214

Cox, Amanda. 2011. "Shaping Data for News," presentation at the 2011 Eyeo Festival. https://vimeo.com/29391942 (quote at 19:29 seconds).

Jeremy Boy, Ronald A. Rensink, Enrico Bertini, Jean-Daniel Fekete. A Principled Way of Assessing Visualization Literacy. IEEE Transactions on Visualization and Computer Graphics, Institute of Electrical and Electronics Engineers, 2014, 20 (12), pp.10.

Robert Kosara, Jock Mackinlay, Storytelling: The Next Step for Visualization, IEEE Computer, vol. 46, no. 5, pp. 44-50, 2013.

Tony Haile. What You Think You Know About the Web Is Wrong. Time. 2014.

http://time.com/12933/what-you-think-you-know-about-the-web-is-wrong/

J. Hullman and N. Diakopoulos. Visualization Rhetoric: Framing Effects in Narrative Visualization. IEEE Transactions on Visualization and Computer Graphics. 2011 
Eden Litt, 2012. Knock, Knock. Who's There? The Imagined Audience. Journal of Broadcasting \& Electronic Media, 56(3), pp.330-345.

Ruggiero, T. Uses and Grats theory in the 21st century. Mass Communication \& Society. 2000, $3(1)$.

B. Shneiderman, C. Plaisant, M. Cohen, S. Jacobs, N. Elmqvist, N. Diakopoulos. Designing the User Interface: Strategies for Effective Human-Computer Interaction (6th Edition). Pearson. 2016.

Ben Shneiderman. The Eyes Have It: A Task by Data Type Taxonomy for Information Visualizations. Proc. IEEE Symposium on Visual Languages. 1996.

Colin Ware. Visual Thinking for Design. Morgan Kaufman. 2008.

Ryan Womack. Data Visualization and Information Literacy. IASSIST Quarterly 2014.

Congressional Budget Office. The 2012 Long-Term Budget Outlook. June 5, 2012.

Acs, Greg. “Responding to Long-Term Unemployment," Urban Institute Report, 2013.

Schwabish, Jonathan. Better Presentations: A Guide for Researchers, Scholars, and Wonks. New York: Columbia University Press. 2016.

Schwabish, Jonathan. "Style Guides," blog post, https://policyviz.com/2016/11/30/style-guides/, November 30, 2016.

CSPAN. "Long-Term Budget Outlook," video https://www.c-span.org/video/?306443-1/longterm-budget-outlook June 6, 2012.

Wiseman, Andrew. "When Maps Lie," CityLab http://www.citylab.com/design/2015/06/when-maps-lie/396761/, June 25, 2015.

Fanny Chevalier, Romain Vuillemot, Guia Gali. Using Concrete Scales: A Practical Framework for Effective Visual Depiction of Complex Measures. IEEE Transactions on Visualization and Computer Graphics, Institute of Electrical and Electronics Engineers, 2013, 19 (12), pp.24262435.

Kim, YS, Hullman, J., and Agrawala, M. Generating Personalized Spatial Analogies for Distances and Areas. ACM CHI 2016.

Huron, S., Vuillemot, R., \& Fekete, J. D. Visual sedimentation. IEEE Transactions on Visualization and Computer Graphics, 19(12), 2446-2455. 
Huron, S. Vuillemot, R. Fekete. J.D. Bubble-TV: Live Visual Feedback for Social TV Broadcast. ACM CHI 2013 Workshop : Exploring and enhancing the user experience for television, Apr 2013, Paris, France.

Jon Huang and Aron Pilhofer, The Death of a Terrorist: A Turning Point? The New York Times (2011) http://www.nytimes.com/interactive/2011/05/03/us/20110503-osama-response.html

Yea-Seul Kim, Katharina Reinecke, Jessica Hullman, Explaining the Gap: Visualizing One's Predictions Improves Recall and Comprehension of Data, ACM Human Factors in Computing Systems (CHI), 2017

Shan Carter, Amanda Cox, Kevin Quealy, The Jobless Rate for People Like You, The New York Times (2009)

http://www.nytimes.com/interactive/2009/11/06/business/economy/unemployment-lines.html

Shan Carter, Kevin Quealy, Home Prices in 20 Cities, The New York Times (2014) https://nyti.ms/2kMCid7

Chris Canipe, Katie Marriner, Stuart A. Thompson, Andrew Van Dam, Beat the Fed, The Wall Street Journal (2016)

http://www.wsj.com/graphics/beat-the-fed/

Uta Keseling, Max Boenke, Reto Klar, Julius Tröger, Christopher Möller, David Wendler, Moritz Klack, Wissen Sie noch, wo Deutschland geteilt war? (Do you still remember where Germany was separated?) Berliner Morgenpost, 2015

http://einheitsreise.morgenpost.de

Office for National Statistics (ONS), Teenage pregnancies - perception versus reality (2016) http://visual.ons.gov.uk/teenage-pregnancies-perception-versus-reality/

Gregor Aisch, Amanda Cox, Kevin Quealy, You Draw It: How Family Income Predicts Children's College Chances, The New York Times (2015)

https://nyti.ms/2jX8zue 